\title{
The anisosphere as a new tool for interpreting Foucault pendulum experiments. Part I: harmonic oscillators
}

\author{
René Verreault ${ }^{\mathrm{a}}$ \\ Université du Québec à Chicoutimi, 555 Boulevard de l’Université, Saguenay, QC G7H 2B1, Canada
}

Received: 2 September 2016 / Received in final form: 30 June 2017 / Accepted: 30 June 2017

(c) The Author(s) 2017

\begin{abstract}
In an attempt to explain the tendency of Foucault pendula to develop elliptical orbits, Kamerlingh Onnes derived equations of motion that suggest the use of great circles on a spherical surface as a graphical illustration for an anisotropic bi-dimensional harmonic oscillator, although he did not himself exploit the idea any further. The concept of anisosphere is introduced in this work as a new means of interpreting pendulum motion. It can be generalized to the case of any two-dimensional (2-D) oscillating system, linear or nonlinear, including the case where coupling between the 2 degrees of freedom is present. Earlier pendulum experiments in the literature are revisited and reanalyzed as a test for the anisosphere approach. While that graphical method can be applied to strongly nonlinear cases with great simplicity, this part I is illustrated through a revisit of Kamerlingh Onnes' dissertation, where a high performance pendulum skillfully emulates a 2-D harmonic oscillator. Anisotropy due to damping is also described. A novel experiment strategy based on the anisosphere approach is proposed. Finally, recent original results with a long pendulum using an electronic recording alidade are presented. A gain in precision over traditional methods by $2-3$ orders of magnitude is achieved.
\end{abstract}

\section{Nomenclature}

(...) KO's original symbols in this

$a, b \quad$ Semi-major and semi-minor axes of elliptical orbit; $b>0$ : counterclockwise (ccw) ellipse; $b<0$ : clockwise $(\mathrm{cw})$ ellipse

$\left(D_{t}^{\prime}\right) \quad$ KO: phase lag after elapsed time $t$

$L, R \quad$ ccw and cw circular states on the anisosphere

$M, N \quad$ Fast and slow elliptical eigenstates

$\left(P^{\prime} L^{\prime} Z^{\prime}\right) \quad \mathrm{KO}$ : spherical triangle in the thesis between longitude great circle, time great circle and characteristic great circle

$\left(p^{\prime}, q^{\prime}\right) \quad$ KO: slow and fast rectilinear eigenstate frequencies

$\left(T_{0}, T_{1}, T_{2}\right) \quad \mathrm{KO}$ : times at start, after first sub-period, after second sub-period

$T_{0}, T_{1} \quad$ This work: average value and first harmonic amplitude of the variation of swinging period vs. swinging azimuth

$T_{X}, T_{Y} \quad$ Swinging period of pendulum for rectilinear eigenstates $X$ and $Y$

\footnotetext{
a e-mail: rverreau@uqac.ca
}

$\left(X, X_{0}, X_{1}, X_{2}\right) \quad \mathrm{KO}$ : azimuths of rectilinear states in general and at the times $\left(T_{0}, T_{1}, T_{2}\right)$

$X, Y \quad$ Fast and slow rectilinear eigenstates on the anisosphere, respectively

$X_{\mathrm{s}}, Y_{\mathrm{s}}$

$\dot{\delta}$

$\dot{\rho}, \dot{\rho}_{F}$

$2 \dot{\rho}$

$\chi$ Stereographic projection coordinates respectively corresponding to $2 \psi, 2 \chi$ on the anisosphere

KO: azimuths of rectilinear slow and fast eigenstates, respectively

KO: initial phase lag between the slow and fast components of a rectilinear oscillation

Rate of increase of phase difference between fast and slow rectilinear eigenstates $X$ and $Y$

$\mathrm{KO}$ : precession rate of a rectilinear state due to Foucault effect Precession rate of a rectilinear state in general, or due to Foucault effect Rate of increase of phase difference between fast and slow circular eigenstates $R$ and $L$ $\mathrm{KO}: \tan ^{-1}(b / a)$ $\tan ^{-1}(b / a)$; half-latitude on the anisosphere 


$\begin{array}{ll}\left(\psi^{\prime}\right), \eta \quad & \text { KO and this work: } \cot ^{-1}(\dot{\delta}) ; \\ & \text { KO: }\left(=\chi_{\max }^{\prime}+\chi_{\min }^{\prime}\right) ; \\ & \text { This work: latitude of slow elliptic } \\ & \text { eigenstate } N \text { on the anisosphere } \\ & \text { Azimuth of major axis of an elliptical orbit } \\ & \text { measured ccw from North; } \\ & \text { half-longitude on the anisosphere } \\ & \text { measured ccw about the upper pole } L \\ & \text { KO: azimuth of the center of a } \\ & \text { sub-period, being at the same time } \\ & \text { the azimuth of an anisotropy axis } \\ & \text { Azimuth of slow eigenaxis of a rectilinear } \\ & \text { orbit in Figure } 17 ; \text { corresponds to } Y \text { on } \\ & \text { the anisosphere } \\ & \text { Frequencies of rectilinear eigenstates } \\ & X \text { and } Y\end{array}$

\section{Introduction}

New interest in a nicely functioning Foucault pendulum may be revived in preparation for the Great American Eclipse on 21st August 2017. Anomalies in the response of many types of pendula, particularly during solar eclipses, have already been reported by different researchers during the last 60 years [1-12]. However, most attempts to reproduce those observations have failed. Needless to say, successive eclipses always occur under different conditions of celestial environment. This makes the task of replicating experiments more complicated. On the other hand, there has not yet appeared a Foucault pendulum standardized in such a way that its response to the surrounding potential could be prescribed. Most of the time, the physical and environmental parameters recorded differ so much that it is not generally possible to conclude on the characteristics of the pendulum used, especially the inherent anisotropy parameters which drastically govern the response.

From Huygens' time, the one-dimensional (1-D) pendulum and coupling between neighboring 1-D pendula have extensively been studied [13]. In two-dimensional (2-D) pendula, a new problem arises from the inherent anisotropy of the system, since the eigenfrequencies in each dimension are in general different. It all boils down to an intricate combination of two types of anisotropy: circular and linear. The ideal Foucault pendulum is assumed to possess pure circular anisotropy in the form of two different translation speeds along the two clockwise (cw) and counter clockwise (ccw) circular orbits which correspond to the eigenstates of the system $[14,15]$. However, when set into rectilinear oscillation within vertical planes at various azimuths, a real 2-D pendulum always shows, for any given amplitude, a period of oscillation that varies with azimuth (linear anisotropy). If a Foucault pendulum is set into rectilinear oscillation, pure circular anisotropy causes the swinging azimuth to precess cw or ccw, the oscillation remaining essentially rectilinear to the naked eye, while pure linear anisotropy will show up as a tendency for the rectilinear orbit to become elliptic and even possibly circular.
At this point, a word should be said about nomenclature. Linear/nonlinear pertaining to pendulum differential equations designates oscillators. A 2-D linear oscillator is a harmonic oscillator which normally has two distinct eigenfrequencies and may have two distinct viscous damping constants. Pendula operating at atmospheric pressure with amplitudes over $1^{\circ}$ are definitely not linear oscillators. Both the restoring torque and the main damping torque involve higher powers of the angular coordinates and their derivatives. However the pendulum studied by Kamerlingh Onnes in his dissertation [16] was a good example of a linear oscillator, since it was operated at amplitudes not exceeding $1^{\circ}$, and at low enough pressures to guarantee viscous damping, originating principally from the deformation of the suspension knives instead of from the surrounding gas.

Linear/elliptic/circular pertaining to bob orbit designates orbit polarization, in analogy with linear/elliptic/ circular polarization of light. Since the bob of a spherical pendulum lies on a spherical surface, a stereographic projection of bob orbit on a horizontal plane will conserve all the angles already on the spherical surface containing the bob trajectory. Hence, a trajectory in a vertical plane will appear as a straight line in the stereographic projection, a small circle on the spherical surface will be projected as a circle, and any trajectory in between will be very close to an ellipse in the projection, assuming that the pole of that stereographic projection is the anti-rest point, one pendulum length vertically above the suspension point. With those definitions, by analogy with optics where a propagating medium may show linear birefringence (e.g., quartz for propagation of light along $a$-axis), circular birefringence (e.g., quartz for propagation of light along $c$-axis) or in general elliptic birefringence when both are present (e.g., quartz for propagation of light along any arbitrary direction), Foucault pendula may also show linear anisotropy (two rectilinear eigenstates swinging at $90^{\circ}$ to one another), circular anisotropy (two circular eigenstates respectively with ccw and cw circular orbits as seen from the suspension point) or in general elliptical anisotropy when both types are present. In this latter case, the eigenstates are two elliptical orbits with the same ellipticity, with their major axes at $90^{\circ}$ to each other and with opposite senses of bob travel along the ellipse.

Among the numerous serious experimenters who have claimed anomalous behavior of their pendula, none of them succeeded in convincing the scientific community that they had sufficiently controlled all the parameters that presumably influence a spherical pendulum. In 1879, despite the very high skill demonstrated in implementing his pendulum setup, Kamerlingh Onnes [15] could not, by design, reproduce his anisotropy axes to better than $\sim 10^{\circ}$ between successive experiments. In aftermath analysis, those axes appear to wander during the day. In the fifties, Allais $[4,5]$ designed two high quality pendula, the first one anisotropic by design (linear anisotropy) and the other one allegedly isotropic. After experimenting for 6 years and finding statistically significant anomalies related to alignment of celestial bodies, his work was brought into controversy and finally dismissed by the French Academy 
of Sciences. Oddly enough, both scientists became Nobel laureates for other work later in their career.

Except for the above two persons who stand aside by the scope and the quality of their work, insofar as recent Foucault pendulum experiments are concerned, too little or no information is given about instrument calibration, anisotropy axis determination, detailed environmental conditions, etc. It remains impossible to scientifically assess such experiments and to distinguish between normal and anomalous response of those various pendula reported in the literature. On the other hand, many contestants of anomalies allege such possible causes as air drafts, rapid atmospheric pressure changes, etc., but, to the author's knowledge, neither has ever shown in which way such phenomena could quantitatively influence a Foucault pendulum.

The present work is to be considered as part I of an operational description of Foucault pendulum response to the non-symmetric potential well that surrounds it. The new formalism of the anisosphere is introduced. It is first applied in aftermath to Kamerlingh Onnes' (KO hereafter) original data which will serve as a 2-D harmonic oscillator test bench for the anisosphere approach. Incidentally, $\mathrm{KO}$ was confronted with a problem which also falls under linear effects, namely the differential damping coefficients of his orthogonal suspension knives (the analogue of dichroism in optics). The special anisotropy induced from that mechanical dichroism is analyzed using the anisosphere formalism. Finally, an operational method of using spherical pendula in the search for anomalies is proposed. It is illustrated with the help of some recent original data.

Part II, to be published separately, will address nonlinear effects. Airy precession [17] is modeled on the anisosphere. Since Allais' highly nonlinear pendula were by design based on the exploitation of Airy precession, the anisosphere sheds a new light on the controversial results that seemed to be correctly interpreted only by their author. The particular procedure of enchained experiments introduced by Allais will also be discussed in part II.

\section{Graphical representation of Kamerlingh Onnes' theory}

Treating the small differences in angular eigenfrequencies (Foucault effect) and in orthogonal swinging frequencies (linear anisotropy) as perturbations, $\mathrm{KO}$ ends up in part 1 , chapter 2, equations (120 ff) with the following set of equations (N.B.: KO's original symbols have been primed in order to avoid confusion with symbols of the present work):

$$
\begin{aligned}
& \cos 2 X^{\prime} \cos 2 \chi^{\prime}=\cos \psi^{\prime} \cos \varepsilon^{\prime}+\sin \psi^{\prime} \sin \varepsilon^{\prime} \cos 2\left(D_{t}^{\prime}+\delta^{\prime}\right) \\
& \sin 2 X^{\prime} \cos 2 \chi^{\prime}= \pm \sin \varepsilon^{\prime} \sin 2\left(D_{t}^{\prime}+\delta^{\prime}\right)
\end{aligned}
$$

where $X^{\prime}$ is the azimuth of the major axis $a$;

$$
\tan \chi^{\prime}=b / a, \quad \text { the axis ratio, }
$$

$\cot \psi^{\prime}=-\left(q^{\prime}-p^{\prime}\right) / 2 \gamma^{\prime}$ is the ratio of the difference in linear eigenfrequencies over twice the Foucault precession rate;

$$
D_{t}^{\prime}=\left[\left(q^{\prime}-p^{\prime}\right)^{2}+\left(2 \gamma^{\prime}\right)^{2}\right]^{\frac{1}{2}} t
$$

designates the time dependent phase of the characteristic circle travel along the time circle at a rate $D_{t}^{\prime} / t$ given by the two rates of linear and circular phase changes combined in quadrature.

$\varepsilon^{\prime}$ is a constant angle determined by the values of $\psi^{\prime}$, $X_{0}^{\prime}$ and $\chi_{0}^{\prime}$.

The geometrical significance of those equations is demonstrated in Figure 1, which reproduces Figure 2 of plate I in the dissertation. Figure 2 (this work) is a remake of KO's Figure 2, in such a way that the great circles have been extended over the complete sphere. In the spherical triangle $P^{\prime} L^{\prime} Z^{\prime}$ between the longitude great circle $\mathrm{L}^{\prime}$, the time great circle $Z^{\prime}$ and the characteristic great circle $K^{\prime}$, the variables pertain to the initial conditions of a pendulum launched into an elliptical orbit. The colors of angles and opposite arcs have been matched, for clarity. $X_{0}^{\prime}$ is the initial azimuth of the major axis; $\tan \chi_{0}^{\prime}=$ $(b / a)_{0} ; \delta^{\prime}$ is the initial phase lag between the components of the initial oscillation along the two rectilinear eigenstates. That graphic representation establishes a unique correspondence between the set of all the sequential oscillating states of the pendulum as time evolves and a family of characteristic great circles that cross the time circle at the constant angle $\varepsilon^{\prime}$ and are generated by turning the initial characteristic circle like a 3 -D object around the rotation axis $M N$ normal to the plane of the time circle.

Summing up, the behavior of a given pendulum setup can be assessed by looking at the spherical triangle $P^{\prime} L^{\prime} Z^{\prime}$. The acute angle near point $P^{\prime}$ gives information about the ratio of linear to circular anisotropy. The smaller that angle, the more Foucault-like the behavior is. A large angle at $P^{\prime}$ is indicative of much linear anisotropy. At any instant, i.e., for any given arc length along the time circle, the arc length along the longitude circle is twice the azimuth of the ellipse major axis with respect to the $X$-axis (Fig. 3 ).

Similarly, the complement angle $2 \chi^{\prime}=2 \arctan (b / a)$ of the angle facing the arc of time circle in the $P^{\prime} L^{\prime} Z^{\prime}$ triangle yields the information about the axis-ratio of the elliptical orbit. Although the above graphical analogue was not exploited any further by KO, it leads the way to a much simpler graphical representation for the motion of a 2-D pendulum.

Incidentally, it is worth mentioning that the geometrical representation of equation (1) by spherical trigonometry has nothing to do with the fact that they describe a spherical pendulum. Strictly speaking, the potential well of a perfect pendulum is spherical and leads to nonlinear differential equations, which will be the subject of part II of this work. KO's treatment deals rigorously with the paraboloidal potential of the harmonic oscillator. It only applies to the pendulum in so far as the latter, in the low amplitude limit, can be approximated by a harmonic oscillator. 


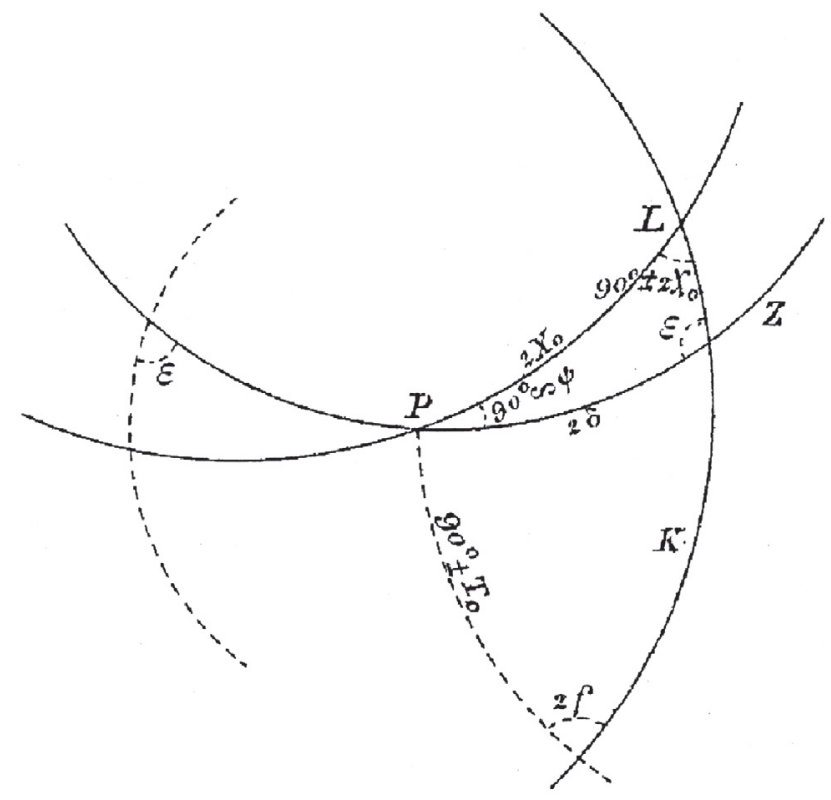

Fig. 1. Graphical representation of KO's equations of motion for a 2-D harmonic oscillator. In the spherical triangle $P L Z$ between the longitude great circle $L$, the time great circle $Z$ and the characteristic great circle $K$, the variables pertain to the initial conditions of a pendulum launched into an elliptical orbit (N.B.: KO used a tilde symbol in front of $\psi$ to replace \pm or $\mp$ according to particular experimental conditions). $X_{0}$ is the azimuth of the major axis; $\tan \chi_{0}=(b / a)_{0} ; \delta$ is the initial phase lag between components along the eigenstates; $\cot \psi=$ $-(q-p) / 2 \gamma$, i.e., the ratio of linear anisotropy to circular anisotropy, expressed in terms of the rates of phase lag between eigenstates; $\varepsilon$ is a constant angle determined by a combination of the initial ellipticity and the anisotropy ratio.

\section{The concept of anisosphere}

The anisotropy parameters included in equations (1) and (3) have been incorporated into Figure 2. They consist of angular velocity vectors proportional to the rates of increase of the phase difference between the two components of any oscillation resolved along the appropriate eigenstates: left-handed $(L)$ and right-handed $(R)$ circular orbits for pure circular anisotropy, or rectilinear oscillations along the $X$ - and $Y$-axes for pure linear anisotropy.

More precisely, the rate of progression of the characteristic circle along the time circle can be seen, according to equation (3), to be governed by an angular velocity vector with magnitude $D_{t}^{\prime} / t$ and normal to the plane of the time circle. It is obviously composed of two orthogonal components. The first one with magnitude $2 \gamma^{\prime}\left(=2 \dot{\rho}_{F}\right.$ later in this work) along the polar axis of the sphere is twice the Foucault precession rate, hence equal to the rate of change of the phase difference between the $\mathrm{cw}$ and the ccw circular eigenstates, the $\mathrm{cw}$ circular state being the faster state in the northern earth hemisphere. With only Foucault effect present, one would have $\psi^{\prime}=90^{\circ}$, and with an arbitrary phase $\delta^{\prime}$ in equation (1), all three circles would coincide. Then $2 \chi^{\prime}=0^{\circ}$ and the minor axis vanishes. In that case, all the points of the equator of

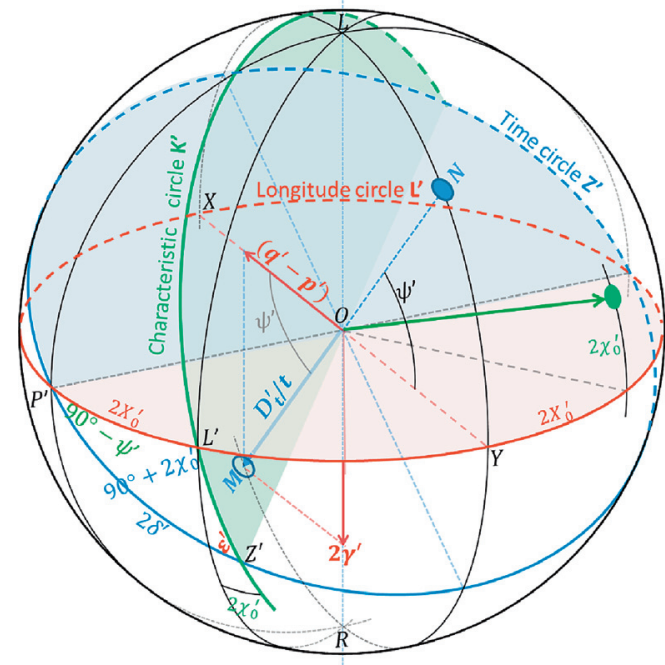

Fig. 2. Extension of Figure 1 on the complete sphere and relation of KO's great circles to the anisotropy parameters. The longitude circle becomes the equator. The circular phase rate of change $2 \gamma^{\prime} \mathrm{rad} / \mathrm{s}$ (twice the Foucault precession rate) is the magnitude of an angular velocity vector pointing towards the lower pole. The linear phase rate of change due to the difference in swinging frequencies, in $\mathrm{rad} / \mathrm{s}$, is the magnitude of an angular velocity vector in the equatorial plane pointing toward the longitude of the faster swinging axis $O X$. The resultant angular velocity vector (in blue) on the diameter $M N$ controls the travel speed of the characteristic circle along the time circle. The normal to the plane of the characteristic circle pierces the surface at longitude $2 X_{0}^{\prime}$ and latitude $2 \chi_{0}^{\prime}$ with respect to the slow axis $O Y$.

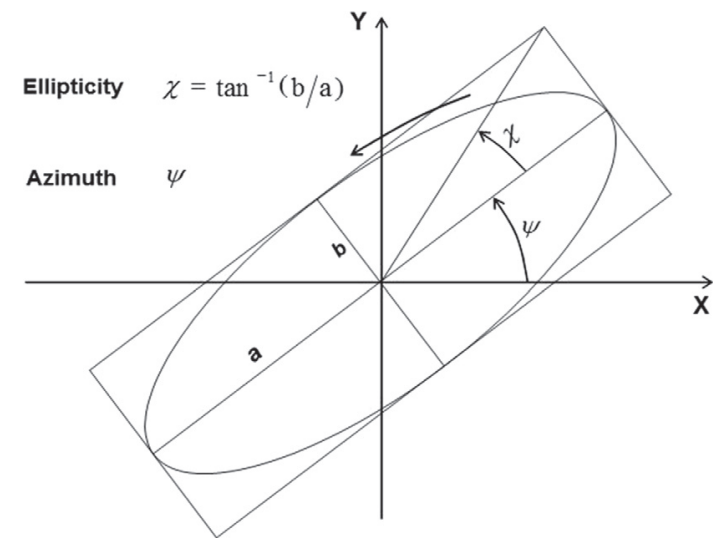

Fig. 3. Proposed nomenclature in this work for the orbit parameters, as seen from the suspension point. $a$ and $b$ are the semi-axes and the angles are positive ccw.

the sphere will represent rectilinear orbits with different azimuths. Let us therefore define the equator of the new anisosphere as the locus of the points representing rectilinear pendulum orbits in such a way that the longitude $2 \psi$ along the equator equals twice the swing azimuth $\left(0<\psi<180^{\circ}\right)$. Contrary to $\mathrm{KO}$ but in accordance with most recent experimenters, $2 \psi$ is counted positive ccw when looking onto the anisosphere from above its north 
R. Verreault: The anisosphere as a new tool for interpreting Foucault pendulum experiments

pole. In this way, orthogonal azimuths parallel to the axes of a laboratory coordinate system $X Y$ will determine on the anisosphere equator two longitudes of diametrically opposed points, $X$ and $Y$.

The second component of angular velocity vector has the magnitude $\left(q^{\prime}-p^{\prime}\right)$ and lies in the equatorial plane, pointing toward the faster rectilinear state $X$. It actually measures the rate of increase of the phase difference between the two components of an oscillation resolved along the rectilinear eigenstates $X$ and $Y$. This designates $X$ and $Y$ as the natural axes of a horizontal coordinate system to measure the swing azimuths, $X$ representing the azimuth of the faster eigenstate, hence with the shortest period. Later in this work $\left(q^{\prime}-p^{\prime}\right)$ will be changed to $\dot{\delta}=\left(\omega_{X}-\omega_{Y}\right)=2 \pi\left(1 / T_{X}-1 / T_{Y}\right)$.

Finally, the resultant angular velocity vector determining the rotation speed along the time circle identifies the rotation axis $M N$ normal to the time circle plane on the anisosphere. From KO's equations, that vector expresses the rate of increase of the phase difference between two elliptical orthogonal eigenstates which can be graphically represented by the diametrically opposed points $M$ and $N$. As for the other cases, the fast eigenstate must be the point $M$ toward which the angular velocity vector points.

Up to now, the new anisosphere has proved capable of representing pairs of orthogonal eigenstates in the form of rotation axes joining two diametrically opposed points of the surface. In fact, such orthogonal eigenstates uniquely characterize individual pendula. Perfect Foucault pendula in the northern earth hemisphere are characterized by the anisosphere polar axis $R L$, the fast state being the cw circular orbit corresponding to the lower pole $\mathrm{R}$ of the anisosphere. Pendula operating on the earth equator should theoretically show pure linear anisotropy and be characterized by a rotation axis $X Y$ in the equatorial plane of the anisosphere, since Foucault effect disappears on the earth equator. In this case, KO's time circle is made up of a meridian/anti-meridian pair defining a plane perpendicular to the $X Y$ diameter on the anisosphere. In general, the double infinity of combinations between the ratios of polar to equatorial components of the angular velocity vector and the azimuths of the $X Y$ equatorial axes correspond to the double infinity of the points on the whole surface of the anisosphere. The anisosphere appears therefore suitable for characterizing any pendulum anisotropy through an appropriate rotation axis $M N$. From usual mathematical conventions, when looking onto the sphere surface from outside, rotation is ccw about the fast state $M$.

Through equation (1), KO has established a correspondence between the oscillation state of a pendulum and a particular characteristic circle, while no simple way to visualize that circle has been provided. But since the plane of the characteristic circle and the plane of the time circle meet under the constant angle $\varepsilon$, the normal to the characteristic circle plane must pierce the sphere surface on a small circle with radius $\varepsilon$ centered on the point $M$, which is the pole of that plane in spherical trigonometry (Fig. 4). Obviously, this small circle is always parallel to KO's time great circle. In order to preserve future development on nonlinear pendula, the name phase circle is proposed. It will be seen later that in nonlinear cases, this circle deforms into a general non circular phase curve where the rate of phase increase is no longer constant. For $\varepsilon<90^{\circ}$ with linear oscillators, the phase circle lies on the faster hemisphere with ccw rotation about the fast eigenstate $M$ as seen from a viewpoint above state $M$. For $\varepsilon>90^{\circ}$, the opposite holds concerning the slow eigenstate $N$.

Looking back at Figure 2, according to KO's results, the acute angle $2 \chi=\tan ^{-1} b / a$ at point $L^{\prime}$ between the characteristic circle and the local meridian must also appear between the characteristic circle normal and the normal to the meridian plane (the latter normal lying in the equatorial plane). Therefore, the latitude of the piercing point of the normal to the characteristic circle bears the information about the axis ratio of the instantaneous pendulum orbit.

Similarly, the intersection line $O P^{\prime}$ between the planes of the characteristic circle and of the equator is perpendicular to the plane containing the angular velocity vectors and the linear anisotropy axis $X Y$. Since $P^{\prime}$ is the origin of the longitudes $2 X^{\prime}$ (after $\mathrm{KO}$ ), the piercing point will have the same new longitude $2 \psi$ with respect to $O X$ as point $L^{\prime}$ with respect to $O P^{\prime}$. Consequently, the piercing point on the anisosphere shows the same azimuth as the azimuth of the orbit major axis.

In summary, it is first possible with the new anisosphere to represent all the possible oscillation states of a pendulum by associating the azimuth $(-\pi<\psi \leq \pi)$ to the longitude $(-2 \pi<2 \psi \leq 2 \pi)$ of a representative point on the surface and the ellipticity angle $\chi=$ $\tan ^{-1}(b / a)$ within the domain $(-1 \leq b / a \leq 1)$ to the latitude $(-\pi / 2 \leq 2 \chi \leq \pi / 2)$ of the same representative point. Conversely, every point of the spherical surface can be associated with an oscillation state. That includes trivial points like the upper and lower poles for which no longitude is defined. They correspond respectively to the ccw and cw circular orbits for which no azimuth is defined. Similarly, the points of the equator which have zero latitude correspond to rectilinear oscillations for which ellipticity is zero. Like the upper pole $L$, the whole upper hemisphere is associated with ccw ellipses, while the lower one with negative latitudes is associated with cw ellipses. Accordingly, cw ellipses must be associated with negative values of the minor axis $b$. Orthogonal states having in general their major axes at $90^{\circ}$ to each other and opposite senses of travel in the ellipses are represented by diametrically opposed points on the anisosphere. Since the surface points of the anisosphere and the oscillation states (or orbits) of the pendulum are both structured sets, and since the relation from one set to the other as well as the inverse relation both preserve the structure, the two sets can be declared isomorph.

Secondly, it is also possible to uniquely characterize on the anisosphere the anisotropy of a given pendulum setup by specifying a rotation axis joining the diametrically opposed points $M$ and $N$ which correspond to the two eigenstates that will remain unchanged in the course of time if the pendulum is excited into one of them. If the 


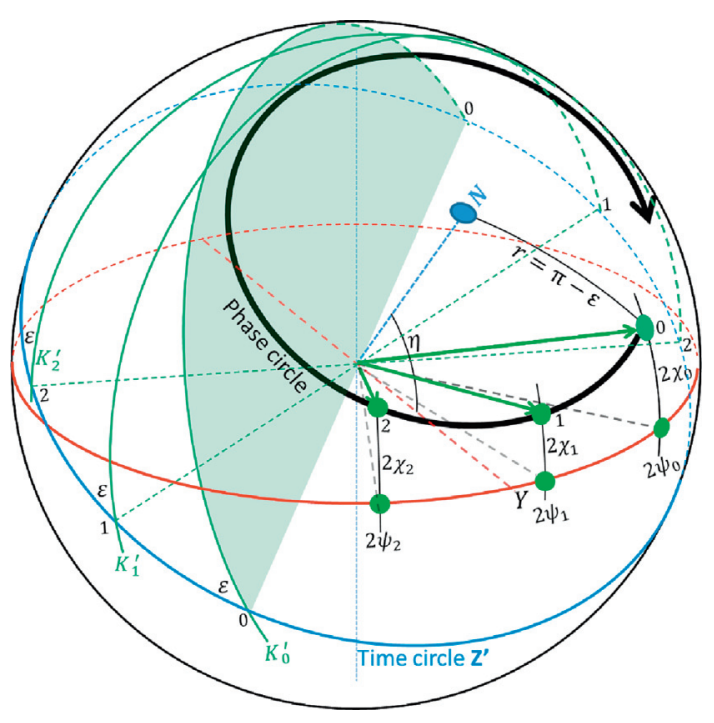

Fig. 4. Determination of the phase circle, i.e., the trace of the pole to the plane of KO's characteristic circle as this one revolves like a solid body about the $M N$ diameter. Numbering 1, 2, 3 designates three successive positions of the characteristic circle with its pole representing the pendulum orbits on the phase circle.

anisotropic character does not change during a pendulum experiment, the position of the rotation axis $M N$ remains constant and the phase curve is a perfect small circle centered either on point $M$ (fast state) with ccw rotation or on point $N$ (slow state) with cw rotation, respectively depending on which one of the fast eigenstate or the slow eigenstate the initial state was closer to, on the anisosphere.

It must be kept in mind that the anisosphere is not exclusive to pendula. It applies to any 2-D oscillating system where a phase difference between the individual components can build up as a function of time or some other variable. Examples can range from Huygens synchronizing double-clocks up to the electric vector of the transverse electromagnetic wave during the propagation of polarized light. This latter case has been dealt with by Poincaré [18]. Using a completely different approach, he described the spatial patterns of polarized light in birefringent crystals due to orthogonal eigenstates of electromagnetic vibration propagating at different speeds. He could uniquely associate to each polarization state along the optical path the ratio of complex amplitudes of both components. The resulting ratios covered the complete complex plane which could then uniquely be mapped onto a sphere (the so-called Poincaré sphere) through stereographic projection. Light is a pure case of $2-\mathrm{D}$ harmonic oscillator where, unlike the time dependence of the pendulum, progression along the phase circle is proportional to the distance travelled in the birefringent medium.

Contrary to Poincaré's original sign convention, the sign convention for the anisosphere is in accordance with a comprehensive application of the Poincaré sphere formalism in optics by Ramachandran et al. in the sixties [19] and with a previous work in magneto-optics by the author [20]. For the azimuths of ellipse major axis referred to the $X$-axis in the $X Y$-plane of the pendulum, ccw sense is counted positively. For the doubled azimuths taken as longitudes on the anisosphere, the positive sense is ccw about the upper pole $L$. When working in the vicinity of the slow axis $Y$, it is customary to use the slow axis as origin for the longitudes, as it is done in Figure 2. Finally, when referring the oscillation azimuth to the geographical coordinates of the earth (what will be called world azimuth), Allais' convention is followed, where the world azimuth is measured positively ccw from north.

\section{Kamerlingh Onnes revisited}

Kamerlingh Onnes' dissertation, written in Dutch, has not been readily available to the English speaking community, except for a few theoretical aspects that have been commented by Schulz-DuBois [14] and for a recently translated chapter on its experimental setup [21]. His aim was to obtain a measure of Foucault precession rate experimentally even by using a highly anisotropic pendulum. By analyzing elliptic orbit evolution, the three independent measurements, namely:

- mean angle between the time great circle and the longitude great circle,

- mean azimuth of the most extreme ellipses,

- repetition period of the ellipse pattern,

yield solutions for the three unknowns:

- azimuths of linear anisotropy axes,

- amount of linear anisotropy,

- amount of circular anisotropy or Foucault rate.

The main construction details significant for the present article are the following:

- a cardan suspension consisting of orthogonal knives articulated in the same plane: an inverted fixed knife bearing the mobile knife structure and the pendulum; the rigid pendulum rod extends above the knives and bears a small round table supporting counterweights in order to adjust the pendulum ellipsoid of inertia,

- double amplitudes ranging from $7 \mathrm{~mm}$ down to $2 \mathrm{~mm}$ measured with a cathetometer; then actual amplitudes in the range $(0.5-2.5 \mathrm{mrad})$ are sufficient for harmonic oscillator behavior,

- an $x y$ coordinate system where the $x$-direction is always that of the fixed knife, which is not necessary an anisotropy axis; the slow anisotropy axis is determined by the azimuth of an extra balancing weight placed near the rim of the round table; in the experiments, one of the anisotropy axis is positioned in the vicinity of the fixed $x$-direction; however in the simulations of plate I, $x$ is used as the azimuth of the slow axis; in KO's thesis, the variable $X$ is an azimuth angle measured with respect to $O x$.

Concerning the anisosphere, azimuths measured in the laboratory with respect to any convenient external reference (e.g., north) are named world azimuths, while the 
azimuth of the normally variable fast axis of the pendulum is called $X$, by definition, on the anisosphere.

That particular value acts as origin for the azimuth $\psi$ of the major axis for any given ellipse.

Although a graphical simulation of KO's theory is presented in plate I at the end of his thesis, no graphical illustration of the experimental results is given. $\mathrm{KO}$ reported 26 meticulous pendulum experiments, but complete sets of data are given only for four of them. He usually conducted his experiments in pairs, first starting the pendulum in the vicinity of the slow anisotropy axis, and then starting it in a similar manner at a $90^{\circ}$ larger azimuth. Since the pendulum is assumed to exhibit a symmetrical behavior near each eigenaxis, KO observed that he could reduce the sources of error by averaging such pairs of experiments, after conversion for major axes of the ellipses at $90^{\circ}$ to each other and for rotations in opposite senses along the ellipses.

\subsection{Pendulum simulations by Kamerlingh Onnes}

Figure 5 shows in the $x y$-plane a sequence of nine orbits calculated by $\mathrm{KO}$ at equal time intervals for an experimental situation classified as B-II. In this class, both ccw and cw ellipses are present, but precession goes in the antiFoucault sense at least for part of the orbit pattern cycle. The value of $\psi^{\prime}<45^{\circ}$ is indicative of predominant linear anisotropy $\omega_{X}-\omega_{Y}>2 \dot{\rho}$, i.e., the difference in rectilinear eigenfrequencies is greater than twice the Foucault precession rate. The particular value $\varepsilon=135^{\circ}$ is determined by the azimuth $X_{0} \approx 17^{\circ}$ of the initial rectilinear oscillation, labelled 0 . The first two $\mathrm{cw}$ ellipses are followed by a rectilinear oscillation again, in $\mathrm{KO}$ nomenclature at $X_{1} \approx-X_{0}$. That marks the end of what he names the normal sub-period (normale onderperiode). Then the abnormal sub-period starts with ccw ellipses, and shortly after, precession goes backwards until shortly before the end of that second sub-period with orbit 8 returning to a rectilinear state identical with the initial one at $X_{2}=X_{0}$. In general, the two sub-periods are delimited by the time of appearance of rectilinear oscillations. For cases where the pattern period involves only ccw or only cw ellipses, the eventual sub-periods are delimited by the flipping time of precession rate.

In this work, a computer model of KO's theory has been elaborated. The ellipses calculated by $\mathrm{KO}$ are obtained using a phase period (period of orbit pattern) $T_{\mathrm{K}}=$ $11.1 \mathrm{~h}$. The model output for the ellipticity angle versus major axis azimuth is shown in Figure 6a. As in Figure 5, one can indeed observe that the ellipse orientations are very close for the orbits 2 and 5 , as well as for the orbits 6 and 7 . Although that graph gives a fair appreciation of the instantaneous precession rate, it is not particularly useful as a diagnosis tool for a given linear to circular anisotropy ratio.

Figure 7 shows the same results using the anisosphere, where the longitude is twice the major axis azimuth and the latitude is twice the ellipticity angle. The representative points for the nine orbits are now evenly distributed (a)

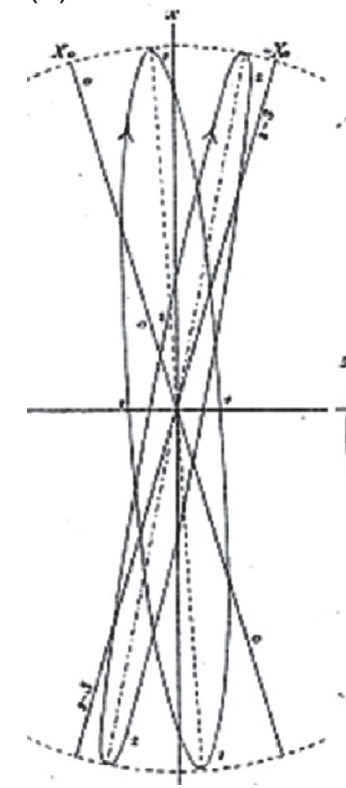

(b)

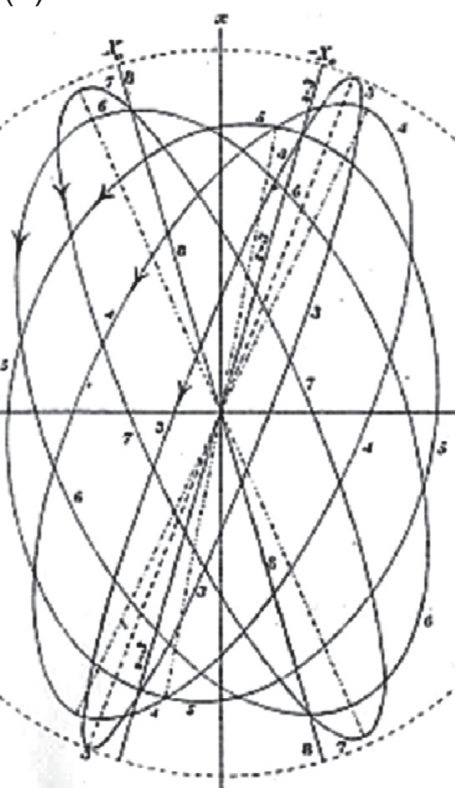

Fig. 5. Composite picture from Figures 5 and 6 in KO's thesis where $\psi^{\prime}=30^{\circ}$ and $\varepsilon=135^{\circ}$, a situation classified by him as B-II. Linear anisotropy amounts then to $\sqrt{3}$ times the Foucault circular anisotropy in that example. Note that ellipses 2 and 5 have practically the same azimuth, and the same holds for ellipses 6 and 7. (a) Normal sub-period, (b) abnormal subperiod.

along the phase circle. The cotangent of the latitude of center $N$ of the phase circle is precisely the ratio of linear to circular anisotropy amounts. Finally, the longitude of $N$ is twice the azimuth of the slow eigenaxis $Y$. In order to convince oneself of the exact shape of the trajectory on the anisosphere described by the representative point of the ellipses, its stereographic projection is given in Figure $6 \mathrm{~b}$. The adjusted circle fits the data perfectly, thus justifying the nomenclature phase circle. Note that the points are not evenly spaced in that figure since stereographic projection preserves the shapes and the angles, but not the distances on the anisosphere.

Figures 8-10 illustrate a case of KO class B-I, with the same anisotropy characteristics but with an initial rectilinear state at azimuth $X_{0}=36.3^{\circ}$ which determines the value $\varepsilon=105^{\circ}$. On the anisosphere, the phase circle starts from the equator at the longitude $2 \psi_{0}=72.6^{\circ}$. The center $N$ is still at $30^{\circ}$ latitude and since the arc radius of the phase circle is $75^{\circ}$, the phase circle passes $15^{\circ}$ behind the pole $L$, thus ensuring that the precession always remains in the Foucault sense. Therefore, the cases of class B-I have two normal sub-periods, but with strongly increasing precession rate when the representative point passes near one of the poles. In this particular case, according to Figures $9 \mathrm{a}$ and $9 \mathrm{~b}$, the azimuth undergoes a sudden decrease of $145^{\circ}$ over $33 \mathrm{~min}$ ( $40 \%$ of the time step), which amounts to 22 times the Foucault rate in the Netherlands. On the other hand, it can be noted on the anisosphere of Figure 10 that the rate of phase change between points 5 and 6 remains constant. It is the rapid crossing of many 
(a)
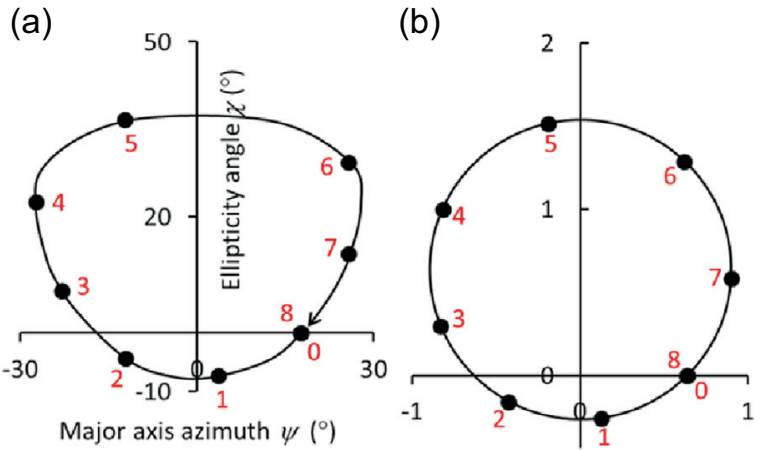

Fig. 6. Computer simulation of the situation of Figure 5. (a) Graph of ellipticity angle as a function of major axis azimuth. Note that the precession rate is much higher for higher values of ellipticity, notwithstanding the fact that the phase difference changes linearly with time. The negative cw ellipses belong to the normal sub-period, always in the Foucault sense. The positive ccw ellipses belong to the abnormal sub-period. On this graph, the fortuitous azimuthal proximities for pair 2-5 and for pair 6-7 are observed. (b) Stereographic projection of the eight points that represent the same ellipses on the anisosphere of Figure 7 . The perfect circle fit in that projection means that the nine points effectively lie on a small circle on the anisosphere.

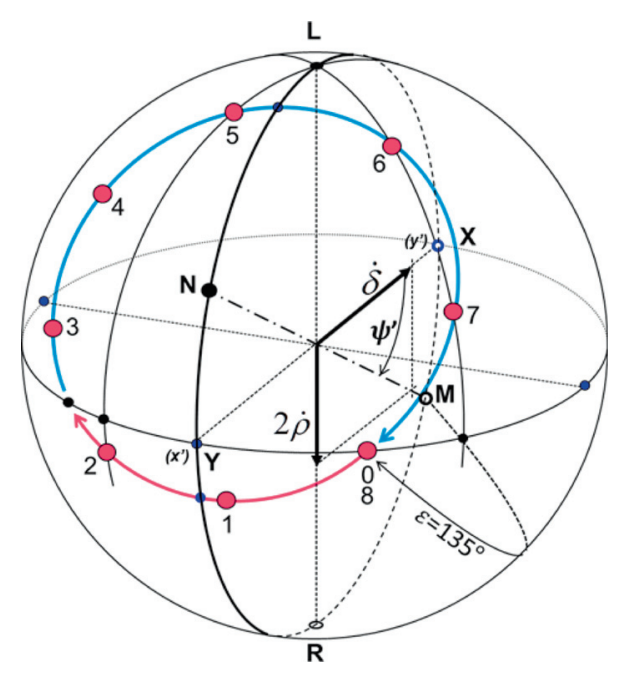

Fig. 7. Perspective view of the nine orbits of Figure 5 on the anisosphere. $\varepsilon$ is the great arc distance from fast eigenstate $M$ to point 0 representing the initial pendulum state. The orbit representative point travels $\mathrm{cw}$ at constant angular speed on the phase circle. The radius of the phase circle about the slow eigenstate $N$ is a great circle arc equal to $\pi-\varepsilon=45^{\circ}$. The phase period (or pattern period) comprises one normal sub-period (in red), monotonously in the Foucault sense, and one abnormal sub-period (in blue) where precession is against Foucault sense for a part of its duration.

meridians that are crowded near the pole which is responsible for the increased precession rate.

The above two simulations give only a partial picture of the power of the anisosphere for visualizing extreme pendulum behavior. It will be shown hereafter, thanks to the anisosphere analysis, that KO's thesis was by far a better pendulum study than he himself could appreciate.

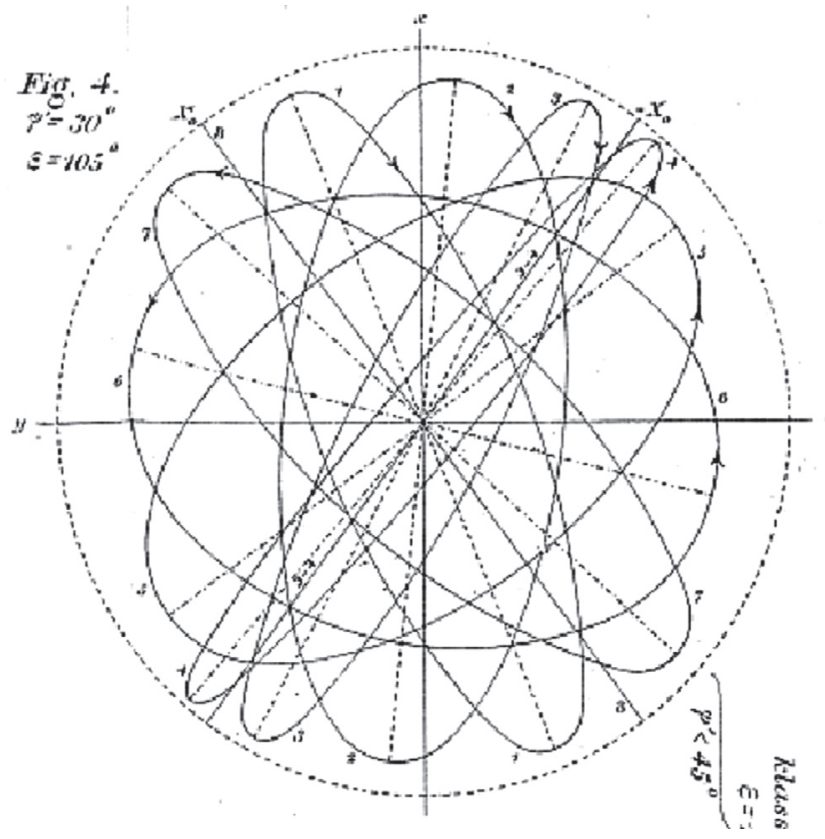

Fig. 8. Reproduction of Figure 4 in KO's thesis where $\psi \prime=30^{\circ}$ and $\varepsilon=105^{\circ}$ a situation classified by him as B-I. There is no abnormal sub-period in that class, although the orbits are partly cw, partly ccw. (a)

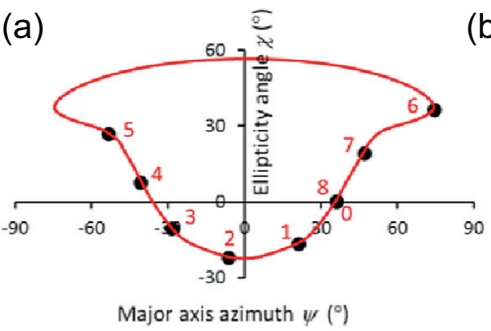

(b)

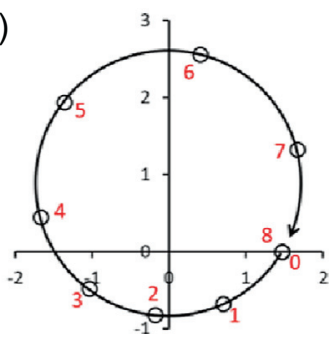

Fig. 9. (a) Graph of ellipticity angle as a function of major axis azimuth for the case of Figure 8. (b) Stereographic projection of the nine points that represents the same ellipses as in (a) on the anisosphere of Figure 10. Despite the somewhat surprising shape of the ( $\chi$ vs. $\psi$ ) graph, the stereographic projection confirms the perfect circle travelled by the orbit representative point on the anisosphere.

\subsection{Pendulum experiment classification}

From now on, some symbols used by KO will be dropped in order to avoid confusion with more recent work. This goes for the linear to circular anisotropy ratio, where $\psi^{\prime}$ of Figures $7-10$ becomes $\eta$ in this work:

$$
\cot \psi^{\prime}=\frac{\omega_{X}-\omega_{Y}}{2 \dot{\rho}}=\frac{\dot{\delta}}{2 \dot{\rho}}=\cot \eta .
$$

Based on the starting state which determines the angle $\varepsilon$ of Figure 11 and on the linear to circular anisotropy ratio which determines the angle $\eta$, nine experiment classes can be defined. For a given general value of $\eta$, class $E$ consists of only two points on the anisosphere, namely the two eigenstates $M$ and $N$ which leave the initial state invariant 


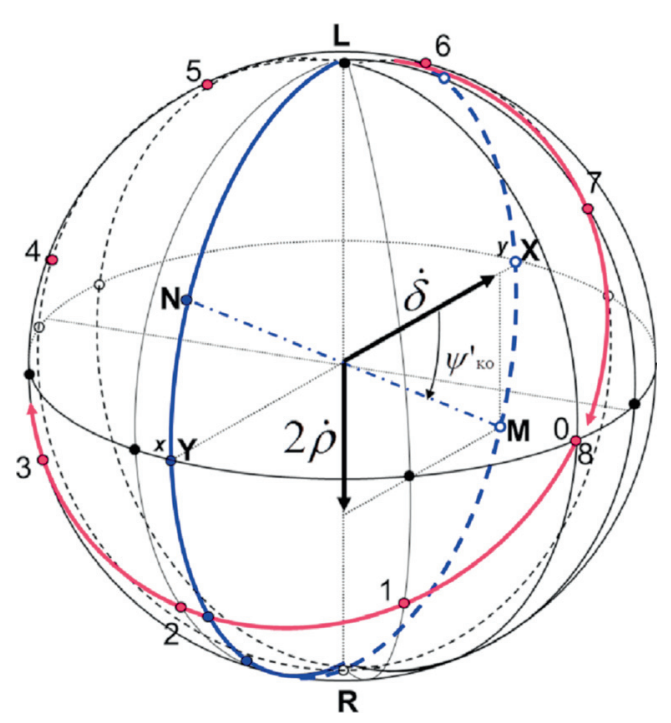

Fig. 10. Representation of the nine orbits of Figure 8 on the anisosphere. The arc radius of the phase circle is $\pi-\varepsilon=75^{\circ}$. Since the phase circle encompasses the pole $L$, precession remains in the Foucault sense at all times.

if the pendulum is started in the elliptical orbit $M$ or $N$. Classes A, D, F-I and F-II consist of circles separating adjacent classes like B-I, B-II and C-I which consist of annular segments of the anisosphere surface or C-II, which is a polar cap centered on one of the eigenstates $M$ or $N$. The mathematical conditions defining each class are as follows:

A: $\cos ^{2} \varepsilon=\sin ^{2} \eta>\cos ^{2} \eta$.

B-I: $\left(\cos ^{2} \varepsilon<\cos ^{2} \eta\right)$ and $\left(\sin ^{2} \eta>\cos ^{2} \eta\right)$.

B-II: $\left(\cos ^{2} \varepsilon>\cos ^{2} \eta\right)$ and $\left(\sin ^{2} \eta<\cos ^{2} \eta\right)$.

C-I: $\left(\cos ^{2} \varepsilon<\cos ^{2} \eta\right)$ and $\left(\sin ^{2} \eta>\cos ^{2} \eta\right)$.

C-II: $\left(\cos ^{2} \varepsilon>\cos ^{2} \eta\right)$ and $\left(\sin ^{2} \eta<\cos ^{2} \eta\right)$.

D: $\cos ^{2} \varepsilon=\sin ^{2} \eta<\cos ^{2} \eta$.

E: $\sin ^{2} \varepsilon=0$.

F-I: $\left(\cos ^{2} \varepsilon=\cos ^{2} \eta\right)$ and $\left(\sin ^{2} \eta>\cos ^{2} \eta\right)$.

F-II: $\left(\cos ^{2} \varepsilon=\cos ^{2} \eta\right)$ and $\left(\sin ^{2} \eta<\cos ^{2} \eta\right)$.

Figure 11 shows how those various classes of experiment are distributed on the anisosphere. For the sake of clarity, only the hemisphere having the slow eigenstate $N$ as a pole is shown. The same classes exist symmetrically in the fast hemisphere having the fast eigenstate $M$ as pole. It is to be understood that class B-I is limited by the two small circles F-I respectively lying in the slow and fast hemispheres. Note that in Figure 11a with $\tan ^{2} \eta<1$, classes A, C-I and F-I do not exist. Similarly, in Figure 11b with $\tan ^{2} \eta>1$, classes B-II, D and F-II do not exist.

In practice, experiments are normally performed by launching the bob into a rectilinear oscillation from a static position away from the pendulum rest point. The initial state is then a point on the equator of the anisoshere, so that the experiment class is either B-I or B-II. There are then two rectilinear states in each phase cycle: the initial one and a symmetrical one on the other side of the slow axis $Y$ or of the fast axis $X$ depending on (a)

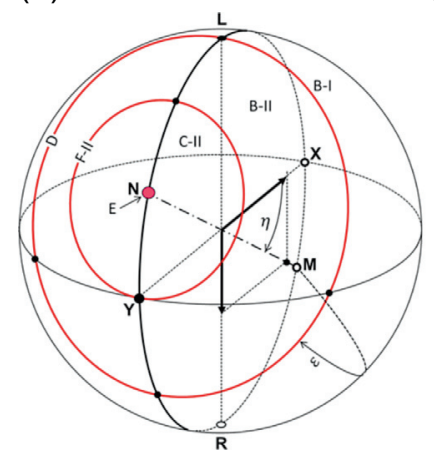

(b)

$\tan ^{2} \eta<1$

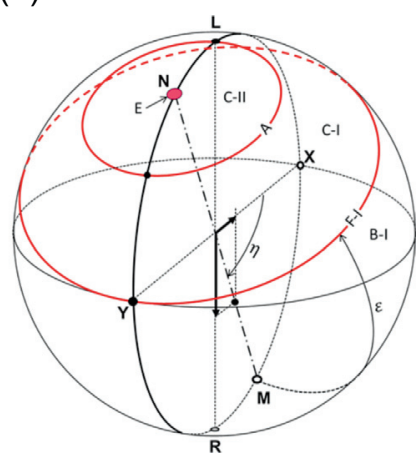

$\tan ^{2} \eta>1$

Fig. 11. Classification of the possible pendulum experiments in the slow eigenstate hemisphere with pole $N$, depending on linear to circular anisotropy ratio and on initial oscillation state. The same classification exists symmetrically in the fast eigenstate hemisphere with pole $M$. Class B-I consists of the symmetrical belt between the fast and slow classes D in (a) or between the fast and slow classes F-I in (b). For the limiting case $\tan ^{2} \eta=1$, classes B-II and C-I disappear and classes A, D, F-I and F-II merge together.

whichever eigenaxis is closest to the initial state. Moreover, there are two sub-periods separated by the rectilinear states. Both sub-periods are normal in class B-I while one of them is abnormal in class B-II.

Exceptionally, one may wish to run an experiment in class F-I or F-II, where the initial state is the only rectilinear state over a complete phase cycle. However, if there is a malfunction during the launch, the experiment might end up accidentally in class C-I or C-II, where the phase circle exclusively consists of elliptical orbits in the same sense.

\subsection{Experimental results - strong anisotropy}

In KO's thesis, the 26 experiments last between $2.5 \mathrm{~h}$ and $3.3 \mathrm{~h}$. Those with complete data sets are experiments A-I, A-II, L-II and M. For the rest of them, only the times marking the sub-period limits and the extreme values of ellipticity angles, the azimuths of those events and finally the major axis amplitude at the sub-period limits are recorded, since they suffice for the determination of the Foucault precession rate.

With the exception of L-I, L-II and M, the experiments were performed in far more anisotropic conditions than the above calculated simulations, in order to be able to observe at least one complete pattern cycle in the $3 \mathrm{~h}$ or so of the experiment duration. Some experiments covered three or four consecutive sub-periods. As mentioned before, they were performed in symmetrical pairs with respect to the fast and slow eigenaxes. A-I was performed near the fast axis and lasted $2.98 \mathrm{~h}$. A-II was performed near the slow axis and lasted $3.21 \mathrm{~h}$.

After the first two experiments, KO declared himself unsatisfied with the behavior of the instrument, because 
(a)

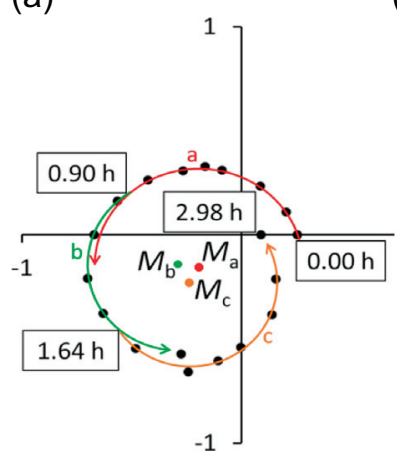

(b)

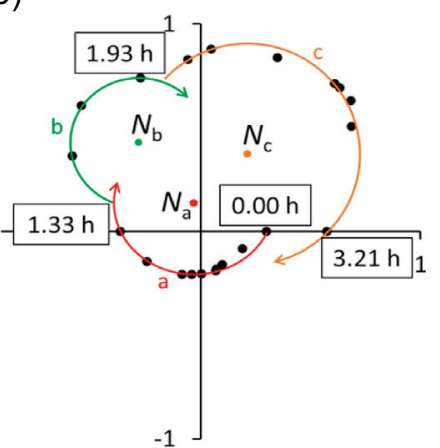

at the coordinates pairs $(2 \psi, 2 \chi)=\left(0^{\circ}, 0^{\circ}\right)$ and $\left(180^{\circ}, 0^{\circ}\right)$ respectively for the fast eigenstate vicinity $M$ and for the slow eigenstate vicinity $N$. The horizontal and vertical coordinates of the projection plane tangent to the equator are:

$$
\begin{gathered}
X_{\mathrm{s}}=\frac{2 \sin 2 \psi \cos 2 \chi}{1+\cos 2 \psi \cos 2 \chi}, \\
Y_{\mathrm{s}}=\frac{2 \sin 2 \chi}{1+\cos 2 \psi \cos 2 \chi} .
\end{gathered}
$$

The reverse transformation is:

$$
\begin{aligned}
& 2 \psi=\sin ^{-1}\left(\frac{4 X_{\mathrm{s}}}{\left(4+X_{\mathrm{s}}^{2}+Y_{\mathrm{s}}^{2}\right) \cos 2 \chi}\right), \\
& 2 \chi=\sin ^{-1}\left(\frac{4 Y_{\mathrm{s}}}{4+X_{\mathrm{s}}^{2}+Y_{\mathrm{s}}^{2}}\right) .
\end{aligned}
$$

This latter transformation is useful for transferring onto the anisosphere the centers of the circles in the projection and to determine the exact position of the rotation axis $M N$ which describes the nature and amount of anisotropy of the pendulum.

It will be also necessary further below (Fig. 14) to use a projection plane tangent to the upper pole of the anisosphere, namely for Foucault pendula with low linear anisotropy where the eigenstates $M$ and $N$ are respectively close to the anisosphere points $R$ and $L$ representing $\mathrm{cw}(R)$ and $\mathrm{ccw}(L)$ circular orbits. In this case, the transformation equations are:

$$
\begin{aligned}
X_{\mathrm{s}} & =\frac{2 \cos 2 \psi \cos 2 \chi}{1+\sin 2 \chi} \\
Y_{\mathrm{s}} & =\frac{2 \sin 2 \psi \cos 2 \chi}{1+\sin 2 \chi} \\
2 \psi & =\tan ^{-1}\left(\frac{Y_{\mathrm{s}}}{X_{\mathrm{s}}}\right), \\
2 \chi & =\cos ^{-1}\left(\frac{4 Y_{\mathrm{s}}}{\left(4+X_{\mathrm{s}}^{2}+Y_{\mathrm{s}}^{2}\right) \sin 2 \psi}\right) .
\end{aligned}
$$

Back to the experimental results, it must first be pointed out that the consecutive data points in Figure 12 show very little scatter, in good agreement with the small measuring errors stated. In fact, three different arcs of circle fit the results very precisely with $95 \%$ confidence level in each experiment for time lapses ranging from 0.6 to $1.5 \mathrm{~h}$. It is therefore observed that KO's law is indeed far better obeyed than its author could himself recognize at that time.

As a matter of fact, KO's calculation of the Foucault precession rate was based on averaging, over A-I and A-II, the initial azimuth, the azimuth of the next passage to a rectilinear state and, for the mid-azimuth $\psi_{D}$ of the respective sub-periods, the minimum ellipticity angle and the maximum ellipticity angle. In doing so, for the second experiment, the sign of ellipticities must be changed and $90^{\circ}$ must be subtracted from the azimuths. Even then, since the azimuth spans of the two sub-periods were not 
R. Verreault: The anisosphere as a new tool for interpreting Foucault pendulum experiments

yet equal, the second sub-period was adjusted equal to the first one and the extreme ellipticity angles corrected by the same percentage. After all those corrections, the four points $\left(\psi_{0}, 0^{\circ}\right),\left(\psi_{D}, \chi_{\min }\right),\left(\psi_{1}, 0^{\circ}\right)$ and $\left(\psi_{D}, \chi_{\max }\right)$ are obtained. Now using the anisosphere analysis, the points $\left(2 \psi_{0}, 0^{\circ}\right),\left(2 \psi_{D}, 2 \chi_{\min }\right),\left(2 \psi_{1}, 0^{\circ}\right)$ and $\left(2 \psi_{D}, 2 \chi_{\max }\right)$ drawn on the anisosphere effectively lie nicely on a small circle, which proves KO's law on the average. However, the anisosphere analysis, through Figure 12, shows that that law is extremely well obeyed already with the raw data, without the above corrections. The corrections actually served only for masking some un-understood phenomenon out of KO's control.

Really, the novel and most important feature of Figure 12 is effectively the occurrence of sudden breaks in the anisotropy character of the pendulum, as marked by the time labels. In each experiment, two anisotropy breaks can be neatly identified.

\subsection{Damping anisotropy or mechanical dichroism}

$\mathrm{KO}$ operated his pendulum in conditions where viscous damping was largely predominant, as shown by Figure 13. Such friction proportional to velocity is a linear phenomenon described by an added term in the linear differential equation of the pendulum, contrary to aerodynamic damping, proportional to $v^{2}$, which is dominant in usual pendulum implementations at atmospheric pressure. Modern pendulum suspensions use flexion of an elastic wire of circular cross-section or rolling of a ball on a polished surface (paraconical pendulum). As such, unless an intentional anisotropic wire is utilized, rolling or flexion losses are presumed isotropic. However, with a cardan suspension, isotropy of losses in the knives may be as hard to achieve as the realization of perfectly isotropic oscillations.

According to the perturbation equations in KO's thesis [22] for the angle $\varepsilon$, the perturbation on the anisosphere due to differential knife friction amounts to a nutation of the rotation eigenaxis $M N$ in phase with the motion of the ellipse representative point on a fast hemisphere phase circle, and at $180^{\circ}$ out of phase with it in a slow hemisphere phase circle. In other words, the eigenstates $M$ and $N$ undergo a small circular motion about their frictionless positions in such a way that the radius of the original phase circle becomes steadily shorter in the fast hemisphere in the vicinity of state $M$, while it gets steadily larger in the slow hemisphere in the vicinity of state $N$. Therefore, the phase curve is no longer a circle but an inward spiral in the fast hemisphere, as shown by the general trend of Figure 12a, and an outward spiral in the slow hemisphere as roughly exemplified by Figure $12 \mathrm{~b}$. So, if an experiment initiated in an elliptic oscillation state close to the slow eigenstate $N$ would last sufficiently long to cover many cycles of the phase curve on the anisosphere, the trajectory of the ellipse representative point would reproduce the path of a right hand knife peeling an apple from top to bottom in one stroke.

It must be kept in mind that $\mathrm{KO}$ had no access to a graphical representation like Figure 12. His analyses

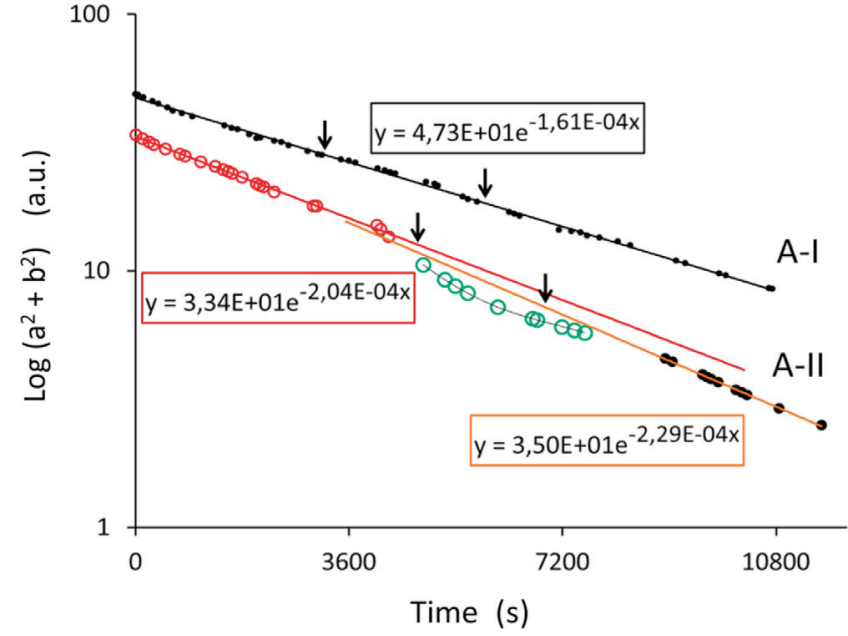

Fig. 13. Damping characteristics of KO experiments A-I and A-II, showing the evolution of the total energy. The arrows mark the times of the anisotropy breaks of Figure 12. On the A-I damping curve, the dot diameter $4 \sigma$ provides an appreciation of the experimental error. In A-II, dot diameter is arbitrary.

were solely based on five special data points: the three times $T_{0}, T_{1}$ and $T_{2}$ where $\chi=0^{\circ}$ and the two extremes values $\chi_{\max }$ and $\chi_{\min }$ at the azimuth $\psi_{D}$. In his words, the above apple analogy was expressed in terms of class mutations, so that an experiment started near the slow axis say, in class C-II (see Fig. 11a) would migrate successively to classes F-II, B-II, D, B-I, D, B-II, F-II, C-II for $\left(\tan ^{2} \eta<1\right)$ or to classes A, C-I, F-I, B-I, F-I, C-I, A, C-II for $\left(\tan ^{2} \eta>1\right)$ (Fig. 11b).

Thus far, there is no definitive clue regarding the cause of the sudden changes in anisotropy of Figure 12. Experiment A-II seems consistent with a mention by KO stating that the sharpness of the knives degraded at different rates during the experiment, thus generating anisotropy from differential damping. The onset of the second anisotropy regime in Figure 12b effectively coincides with the first discontinuity in the exponential damping curve of Figure 13. This could be caused by a sudden rupture in the microscopic shape of a knife edge followed by sporadic crushing of the debris. Finally, exponential damping is resumed but at a new rate and roughly half an hour after the beginning of the third anisotropy regime.

On the other hand, the significant anisotropy changes in Figure 12a bear no apparent relation to global damping, which remains strictly described by a single exponential curve within the very small measuring errors (Fig. 13). Hence, no significant change in damping regime can be detected in A-I. This suggests that some unknown cause other than differential damping may be responsible for those small changes in anisotropy for the pendulum.

An attempt to fit inward and outward spirals respectively to Figures $12 \mathrm{a}$ and $12 \mathrm{~b}$ would leave residuals by far incompatible with the experimental errors. It can be seen though that the eigenstates $M$ and $N$ do wander in the sense predicted by KO friction theory, but they do so 


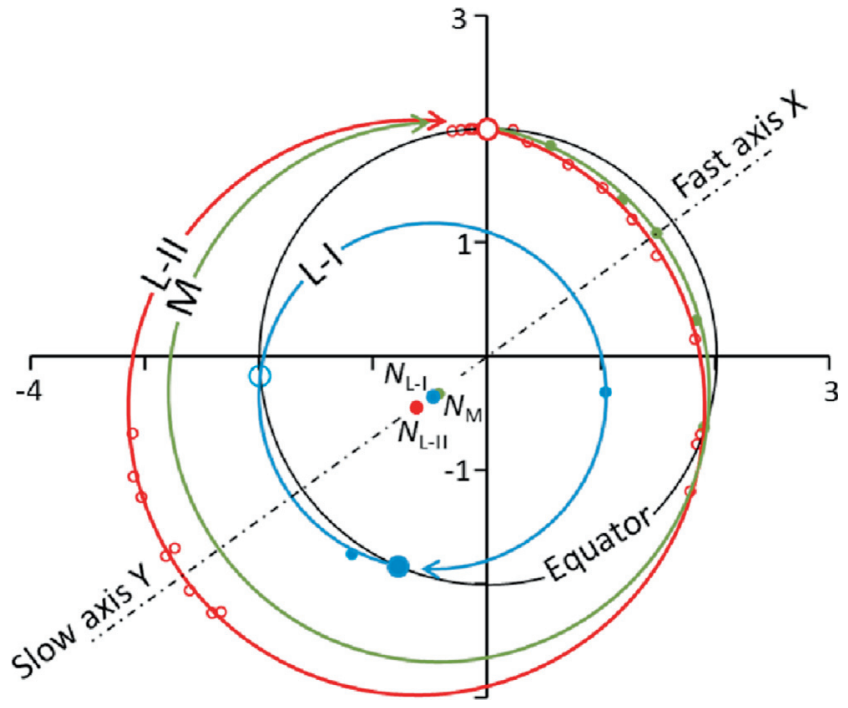

Fig. 14. Experiments L-I, L-II and M in a polar stereographic projection of the anisosphere, the image plane being tangent to the anisosphere at the upper pole. The black circle that crosses the axes at $X_{\mathrm{s}}= \pm 2$ and $Y_{\mathrm{s}}= \pm 2$ (coordinates not shown in the graph) is the projection of the equator. It represents the phase circle of an ideal Foucault pendulum. Circle arcs that lie inside the black circle represent positive, $\mathrm{ccw}$ ellipses while those lying outside represent negative, $\mathrm{cw}$ ellipses. Note that the anisotropy axes are common to the three experiments.

stepwise and stand still in between. More experimentation is necessary to verify the theory of differential damping. However, cardan pendulum suspension seems to have been abandoned by present day experimenters.

\subsection{Experimental results - weak anisotropy}

The last experiments L-I, L-II and $\mathrm{M}$ reported by $\mathrm{KO}$ are conducted with very good compensation of linear anisotropy, thus approaching the ideal Foucault pendulum. The ratio of residual linear anisotropy to Foucault anisotropy is $\sim 30 \%$. This is typical of well-designed long pendula in the height range $6-10 \mathrm{~m}$, which is very good for a $1.4 \mathrm{~m}$ pendulum. These experiments belong to $\mathrm{KO}$ class B-I where no abnormal sub-period is present.

Figure 14 shows a polar stereographic projection of the anisosphere, the image plane being tangent to the anisosphere at the upper pole. The black circle that crosses the axes at $X_{\mathrm{s}}= \pm 2$ and $Y_{\mathrm{s}}= \pm 2$, is the projection of the equator. It represents the phase circle of an ideal Foucault pendulum. Circle arcs that lie inside the black circle represent positive, ccw ellipses while those lying outside represent negative, cw ellipses.

Experiment L-I is somewhat precarious. It is initiated in the neighborhood of the slow axis. The initial rectilinear state is represented by the lowest blue dot at the crossing of the blue phase circle (Fig. 14) and the equator circle. Only the three points shown as blue dots were actually measured; the fourth one (small blue circle) with zero ellipticity was assumed symmetrical to the initial

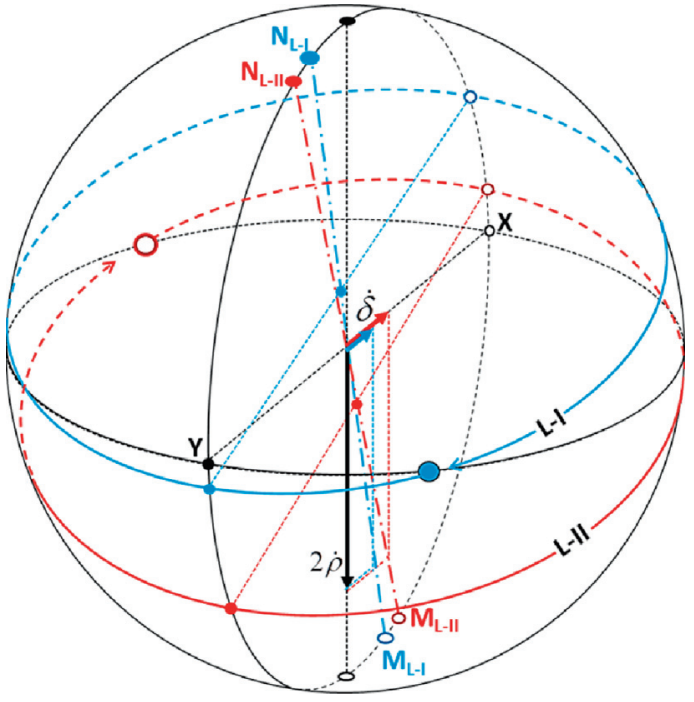

Fig. 15. Illustration of experiments L-I (in blue) and L-II (in red) on the anisosphere. The linear to circular anisotropy ratio $\dot{\delta} / 2 \dot{\rho}=0.32$. For L-I, the initial azimuth (big blue dot on the equator) is closer than $45^{\circ}\left(2 \psi_{0}<90^{\circ}\right)$ to the slow axis $Y$ determined by the meridian plane containing the blue anisotropy rotation axis $M_{\mathrm{L}-1} N_{\mathrm{L}-1}$. With cw rotation about the slow eigenstate $N$, one obtains $\mathrm{cw}$ ellipses in the first sub-period, then ccw ellipses in the second one. Similarly, for L-II, the initial azimuth (big equatorial red dot behind the anisosphere) is closer to the fast axis $X$. One then obtains ccw ellipses in the first sub-period and cw ones in the second sub-period.

state on the other side of the presumed slow anisotropy axis, as theory would dictate, but it was annotated by $\mathrm{KO}$ : niet waargenomen (not observed). That experiment was intended to be paired with L-II and have the same anisotropy characteristics. Hence the blue phase circle should be concentric with the red one, but strict experimental data is not sufficient to support such an assertion. If this were true, the third experimental point on the extreme right should lie on the fast anisotropy axis, since it is presented by $\mathrm{KO}$ as the state with maximum ellipticity. By anisosphere analysis, this is obviously not the case. Notwithstanding the lack of accuracy, the qualitative evolution of the phase circle is correct, showing, with cw travel around the slow eigenstate $N_{\mathrm{L}-\mathrm{I}}$, first cw ellipses toward the slow axis, and then wider ccw ellipses in the vicinity of the fast axis. The perspective view of the anisosphere in Figure 15 allows a better visualization of the situation.

Experiments L-I and L-II were meant to be identical except for an interchange of fast and slow axis vicinity. However, the anisosphere shows that the anisotropy axis remained the same but the amount anisotropy, measured as $\dot{\delta}$ in Figure 15, fell short in L-I by $\sim 30 \%$ of the value realized in L-II. It can be noted in Figure 14 that the blue circle, instead of being concentric with the red circle as it should, is concentric with the green one of experiment M, which was designed with the same anisotropy axis but $30 \%$ less linear anisotropy than experiment L-II. 
R. Verreault: The anisosphere as a new tool for interpreting Foucault pendulum experiments

Experiment L-II, on the other hand, was performed very meticulously in the neighborhood of the fast axis. In Figure 14, the initial state (small red circle) lies at the upper intersection of the red phase circle with the equator circle. $\mathrm{Cw}$ travel around the slow eigenstate $N_{\mathrm{L}-\mathrm{II}}$ generates first ccw ellipses, then a rectilinear state symmetrical with the initial state on the other side of the fast axis. This marks the end of the first sub-period and the start of the second one with the same precession sense but $\mathrm{cw}$ ellipses. In the vicinity of the equator of the anisosphere (Fig. 16), the phase circle is well approximated by the Lat-Lon relation:

$$
2 \chi=\left(\varepsilon-90^{\circ}\right)+\eta \cos \left[2 \pi\left(2 \psi-2 \psi_{X}\right) / 360^{\circ}\right],
$$

where,

$\varepsilon$ is the arc radius of the phase circle from the fast eigenstate $M$ taken as center on the spherical surface (see Fig. 7);

$\eta=\cot ^{-1}\left(\frac{\dot{\delta}}{2 \dot{\rho}}\right)$ is the inverse cotangent of linear to circular anisotropy ratio;

$\psi_{X}$ is the world azimuth of the fast linear anisotropy axis, identified as $\mathrm{X}$ on the anisosphere.

Using the complete experimental information by considering the fitted curve of Figure 16,

$$
\text { and } \begin{aligned}
\varepsilon_{\mathrm{L}-\mathrm{II}} & =\left(80.24^{\circ} \pm 0.17^{\circ}\right), \\
\eta_{\mathrm{L}-\mathrm{II}} & =\left(17.55^{\circ} \pm 0.20^{\circ}\right) .
\end{aligned}
$$

The latter must be compared to,

$$
\eta_{\mathrm{L}-\mathrm{II}}=\psi_{\mathrm{KO}}^{\prime \prime}=18.57^{\circ} \text { and } 17.85^{\circ},
$$

as calculated by KO using respectively the first and second sub-period data. Hence, with the anisosphere and using the same data, pendulum parameters can be determined more precisely than with KO's method.

Summarizing the revisit of KO's thesis, the anisosphere analysis proves to be an extremely efficient tool to study pendulum behavior. Qualitatively, it permits an outstanding visualization of the evolution of pendulum orbits throughout the experiment. This is particularly useful for distinguishing the various classes of experiments that were described through conditions on certain critical angles in KO theory. Quantitatively, by using stereographic projections on planes tangent to the anisosphere either at the equator or at the poles, it is possible to calculate the exact shape of the phase curve on the anisosphere by fitting circle arcs or, in general, any computable shape to the experimental data, since shapes are conserved in that kind of projection. Thanks to the anisosphere analysis, it has been shown that KO theory was verified by his experiments in a far better way than he effectively could appreciate with the limited analysis means at his disposal. Finally, anisosphere analysis allowed the detection of unexpected changes in the anisotropy character of the pendulum within a single experiment (e.g., A-II) or between supposedly identical experiments (e.g., L-I and L-II).

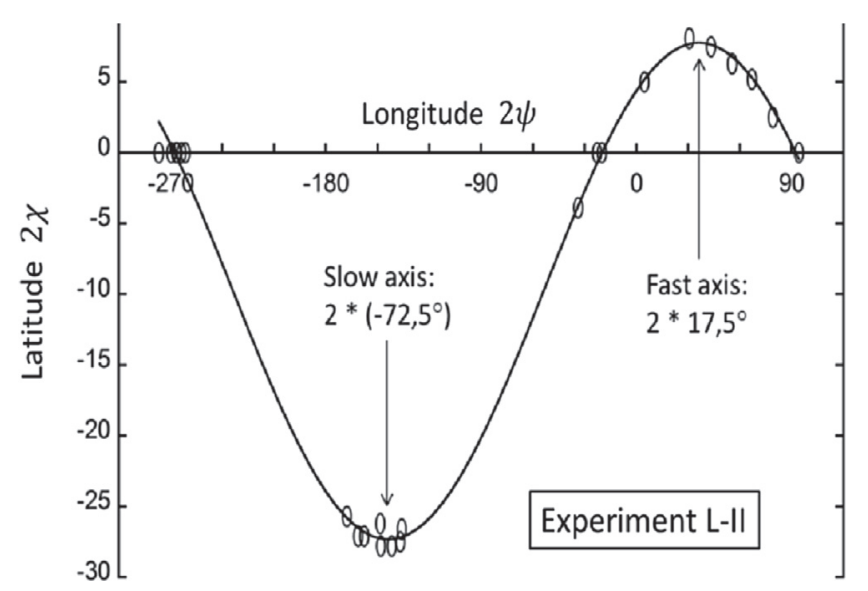

Fig. 16. Least squares fit of a phase circle of the anisosphere to the data of experiment L-II. The vertical dimension of the data point symbols is equal to $2 \sigma_{\text {Lat }}=1.4^{\circ}$. The precession rate being negative, the initial state lies at the extreme right. The fast-(slow-)axis azimuth is given by the maximum (minimum) of the fitted cosine function.

\section{Experiment strategy}

In the last six decades, the numerous experimenters referred to in the introduction above have tried in various ways to look for anomalous behavior of their 2-D pendulum in conjunction with possible perturbing phenomena. In that sense, Allais' direct search [1-3] for a change in the anisotropy characteristics of his pendulum constitutes a pioneering work. Alternately, his followers were rather looking for changes in precession speed or in period of oscillation. However, without stating it explicitly, they were addressing parameters which respond respectively to circular anisotropy or linear anisotropy. Consequently, thanks to the present new approach describing pendulum anisotropy via the anisosphere, pendulum experiments can be conducted with the aim of measuring quantitatively the changes in the ratio of linear to circular anisotropy amounts as well as the changes in the azimuth of the anisotropy axes. The anisosphere analysis shows that a pendulum's behaviour is not determined by the sole amount of linear anisotropy $\left(\omega_{X}-\omega_{Y}\right)$ but by its comparison to $2 \dot{\rho}$, the doubled Foucault precession rate. Hence, an essentially isotropic pendulum at mid-latitudes with $\left(\omega_{X}-\omega_{Y}\right) \ll 2 \dot{\rho}$ may prove readily anisotropic near the equator, whereas the same value of $\left(\omega_{X}-\omega_{Y}\right)$ is much greater than twice the local Foucault rate.

In practice, the circular phase rate of change $2 \dot{\rho}$ due to Foucault effect is easily calculated. Thereafter, the experimental procedure should include the following stages:

(a) an approximate determination of anisotropy axes and linear phase rate of change by conducting a series of short experiments at different azimuths in the range $0^{\circ}-180^{\circ}$, and by measuring the various swinging period values $T$ for rectilinear oscillations. Fitting a cosine function to a graph of period vs. azimuth yields, for the eigenaxis azimuths, those of the period extrema 
$T_{Y}, T_{X}$, and for the amount of linear anisotropy, the value:

$$
\dot{\delta}=\left(\omega_{X}-\omega_{Y}\right)=\frac{2 \pi\left(T_{Y}-T_{X}\right)}{(\bar{T})^{2}}=\frac{8 \pi\left(T_{Y}-T_{X}\right)}{\left(T_{Y}+T_{X}\right)^{2}},
$$

(b) a precise determination of the anisotropy axes by launching an experiment at an azimuth just a few degrees higher than the approximate value of azimuth for that axis, so that Foucault precession will provide an azimuth scan through the minimum latitude of the phase circle for a slow axis, or through the maximum latitude of the phase circle for a fast axis. Again, from fitting a cosine function to a graph of anisosphere latitude vs longitude, a far better estimate of the eigenaxis azimuth is obtained as the azimuth of the latitude extremum.

Ideally, the short experiments of stage (a) should be evenly distributed within the $180^{\circ}$ azimuth domain in order to maximize the statistical significance of the least squares fit of the cosine function. Indeed, Allais [3] has shown that the azimuths of the eigenaxes may vary with time within a day. Therefore, it is best to choose the sequence of launching azimuths at random, so that a temporal bias in azimuth is avoided.

Finally, the value of a period measurement is strongly dependent on amplitude and on temperature, in the range of interest for anisotropy determination. Therefore, care must be taken so that all period measurements be taken at precisely the same swinging amplitude.

In so far as temperature is concerned, the author has already measured temperature variations of $10 \mathrm{~K}$ between day and night near the suspension structure in certain implementations of a pendulum. Steel and most metals that can be used for suspending the bob have a thermal expansion coefficient of the order of $0.00001 \mathrm{~K}^{-1}$, which means an increase in period of $\sim 25 \mu \mathrm{s} / \mathrm{K}$ for a typical pendulum with a period of $5 \mathrm{~s}$. In stage (b), precision is of the utmost importance. Depending on the retrieval method for bob motion, various launching disturbances can occur, some of them fortunately decaying with time. In many cases, a rigid pointer coaxial with the suspension wire extends below the pendulum bob. Its instantaneous position is measured either from visual observation against some kind of alidade or from timings via some sort of electronic device (video camera, optical beam interruption or any convenient type of electronic alidade). Contrary to manual observation, electronic recording can yield parameter values for each individual cycle; it renders efficient computerized data processing possible.

However, among the most annoying perturbations at that precision level, one counts the initial transient nodding of the bob which is caused by misalignment between the pre-start retaining thread and the center of mass of the bob-stem assembly, plus a permanent nodding that is generated by the non-constant returning moment applied by the suspension line at the stem attach point. The stem is a rigid rod between the bob per se and the attach point of the flexible suspension wire. Its role is to provide a lever arm in order to force the bob axis of revolution symmetry to stay aligned with the suspension wire at all times, in spite of the ever changing angular velocity of the bob about a horizontal axis perpendicular to the oscillation plane. The bob-stem unit has its own, rather high, eigenfrequency of angular oscillation about this horizontal axis. The peak returning torque at the end of each half-cycle generates a nodding oscillation which beats with the pendulum oscillation proper and causes aliasing in the recorded bob position. To minimize permanent nodding, the stem must meet a compromise between length, stiffness and small moment of inertia about a horizontal axis. To minimize initial nodding, the correct hook height of the retaining thread to be burnt must be found by trial and error.

Another small mishap which is often seen as a perturbation is a launch before the bob is completely stabilized at the end of the retaining thread. This generates an initial elliptical orbit with unpredictable sense of travel. This is rather difficult to interpret using conventional analysis. However there is really nothing to it when analyzed with the anisosphere. That initial ellipse is forcibly somewhere on the normal phase circle representing that pendulum's evolution. As an example, for an experiment in the vicinity of the slow axis, a ccw ellipse (positive semi-minor axis) means that the initial state lies above the equator of the anisosphere, so that a rectilinear state will soon appear at the start of the first sub-period. Thereafter a situation like the one of Figure 12b follows. If, on the other hand, the initial ellipse is cw, everything will proceed as if the experiment in Figure $12 \mathrm{~b}$ would have started at some time later than zero. That $\mathrm{cw}$ ellipse will grow wider up to an extremal ellipticity at the azimuth of the slow axis, then become thinner up to the rectilinear state at the end of the first sub-period, and so on. Such an experiment will suffer no significant lack of precision in the determination of the slow axis azimuth according to stage (b).

\section{Original experimental results}

The author is a member of a team of designersexperimenters who operate a Pendularium (permanent pendulum site) in Horodnic-de-Jos, Romania. The particulars of that Pendularium will be described elsewhere [23]. Suffice it to mention that pendulum motion is retrieved from a sequence of 16 timings per cycle as a pointer under the bob crosses four narrow beams of light in an M-configuration. As soon as an experiment is over, the following physical parameters are immediately obtained by software for each cycle: period, amplitude, azimuth of major axis, value and sign of semi-minor axis, $x$ - and $y$ coordinates of oscillation center, latitude on the anisosphere and cumulative Airy precession, together with a set of useful diagnosis graphs.

Figure 17 shows a graph of swing period as a function of swing azimuth resulting from a stage (a) experiment described in the preceding section. This graph has the 
R. Verreault: The anisosphere as a new tool for interpreting Foucault pendulum experiments

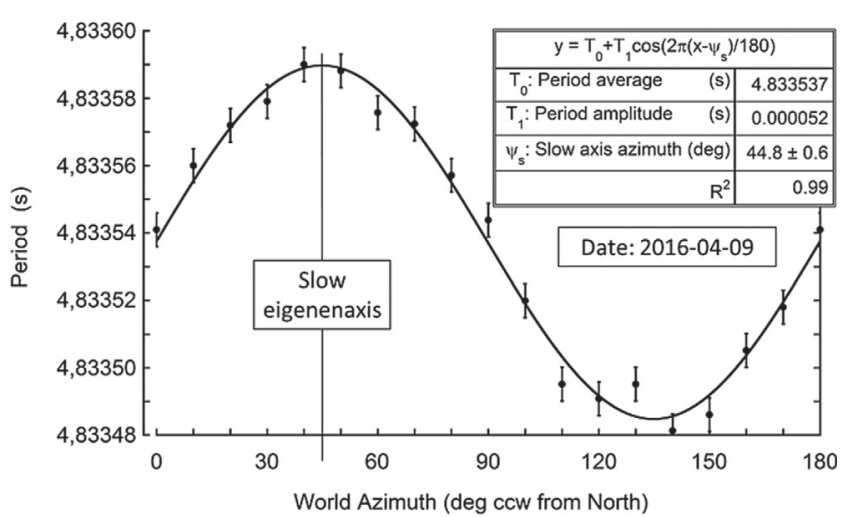

Fig. 17. Period of a Foucault pendulum as a function of swinging azimuth, according to stage (a) of the original experiment of this work. The data are corrected for temperature and temperature gradient along the wire. The values are retrieved by software as an average over a 25-cycle interval centered on a cycle always taken at the same swinging amplitude.

particular merit of giving directly the parameters corresponding to the very definition of pendulum linear anisotropy, namely the difference in extreme frequencies (from the extreme periods) together with the slow and fast eigenazimuths. The standard error of estimate $0.6^{\circ}$ (see insert of Fig. 17) for the eigenaxes is quite sufficient as a guide for designing experiments of stage (b). But above all, this graph is among the most dependable methods of measuring a pendulum's amount of linear anisotropy averaged over the time span necessary for the whole series of short experiments to be completed.

Following the above three-day preliminary stage ending on April 9, 2016, stage (b) consisted of a series of 71 longer experiments all initiated from a rectilinear state at the world azimuth $60^{\circ}$. Figure 18 shows the result of \#53 of that series, realized on April 21.

First of all, the slow axis azimuth of $\left(55.66^{\circ} \pm 0.01^{\circ}\right)$ is clearly quite different from the value $\left(44.8^{\circ} \pm 0.6^{\circ}\right)$ measured on April 7-9. As a matter of fact, without changing anything to the pendulum suspension, a general drift toward higher azimuths has been observed from day to day. Also, within a given day, eigenaxis fluctuations apparently related to the outdoor temperature have been observed. Those phenomena will be published separately. Within the scope of the present work, note that the slow axis azimuth is very accurately obtained by fitting a cosine function to the data around the minimum of the phase circle latitude on the anisosphere (Fig. 18). This method really seems indisputable on the basis of $\mathrm{KO}$ theory, assuming the pendulum to act as a 2-D harmonic oscillator.

Harmonic oscillator behavior is best realized by long pendula at low amplitude, or by short pendula at extremely low amplitude as in KO's thesis. However, real pendula all show nonlinear behavior to a certain extent. As it will be shown in part II of this work, nonlinearity results in a phase curve on the anisosphere that departs from the circular shape. In the example of Figure 18, the consistency of the 681 cycles that led to the close

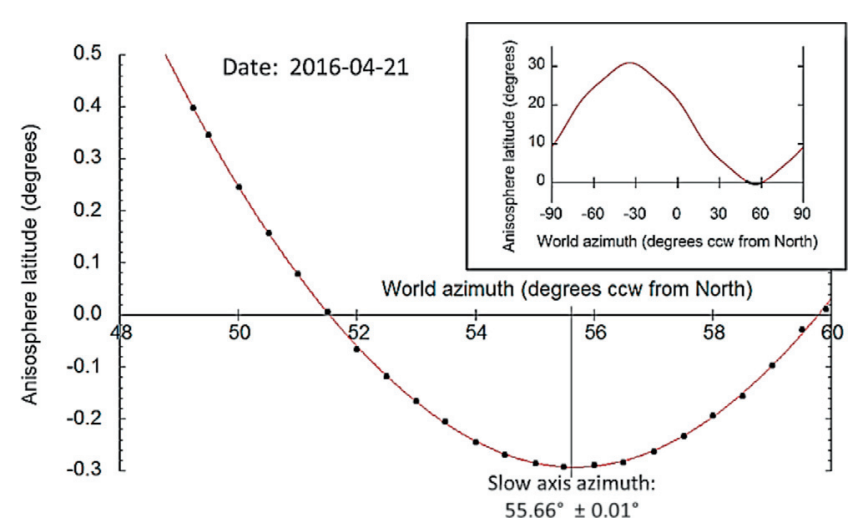

Fig. 18. Adjustment of a cosine function to the experimental data points translated to latitudes on the anisosphere, according to stage (b) of the original experiment of this work. For graph clarity, only one cycle every half degree is shown, although 681 consecutive cycles are included in the curve fitting. The dot diameter represents $4 \sigma$, so that $95 \%$ of the dots make contact with the theoretical curve. The insert shows the equivalent of Figure 16, the extrapolated part of the curve being based on the short interval of measured values between the azimuths $49^{\circ}$ and $60^{\circ}$. Slow axis determination benefits a gain in precision by 2-3 orders of magnitude over KO's method.

fit illustrated there should lead to a phase circle tilted, with respect to the anisosphere equatorial plane, at the angle $\left(90^{\circ}-\eta\right)=\tan ^{-1}\left(\left(\omega_{X}-\omega_{Y}\right) / 2 \dot{\rho}\right)$ from which the amount of linear anisotropy can be obtained. Of course, such huge extrapolation as illustrated by the insert of Figure 18 is not a dependable method of measuring linear anisotropy, contrary to the direct procedure of Figure 17. However, the fit of Figure 18 actually consists of a cosine with a period of $180^{\circ}$ and an amplitude dictated by the result of Figure 17, plus $3.5 \%$ of a single higher harmonic content. In this case, therefore, the phase curve on the anisosphere is a slightly distorted circle. Its departure from the perfect circle becomes a way of assessing the amount of nonlinearity present in the Pendularium setup.

\section{Conclusion}

Based on the equations of motion derived by Kamerlingh Onnes for the 2-D pendulum, the anisosphere has been introduced as a new graphical method to describe and analyze such pendula. This part I of anisosphere description has dealt with harmonic oscillators, a situation corresponding indeed to very few implementations of real pendula, but constituting a passage obligé before addressing the more sophisticated study of nonlinear oscillators. The high performance pendulum built by KO in 1879 as well as some long Foucault pendula at low amplitude are among those that may qualify as linear oscillators. Through re-analysis of KO's thesis using the anisosphere, the outstanding visualizing power of that new approach has been demonstrated. The largely hermetic canonical equations describing Foucault pendulum motion as well 
as the hard to visualize conditions at the origin of the different classes of Foucault pendulum experiments all take a new significance when illustrated on the anisosphere. Thanks to anisosphere analysis, it has been shown that KO theory was far better obeyed in his own experiments than he could himself imagine. Some of his questions about unreliability of certain experimenting situations have received an answer, especially the new discovery of sudden breaks in anisotropy properties of his pendulum in the course of a given experiment.

By representing the anisotropy character of a given pendulum implementation on the anisosphere, a new experiment strategy could be elaborated. The search for anomalies in pendulum behavior when the instrument is submitted to various types of perturbation finally boils down to recording changes in the anisotropy properties of the surrounding pendulum potential well. A very efficient method of measuring the azimuth of the anisotropy axes has been presented. Coupled with modern electronic devices for recording the pendulum physical parameters at each cycle of oscillation, the anisosphere approach permits an exhaustive exploitation of all the redundant recorded information to determine the anisotropy axis position with unprecedented accuracy. In this way, any minute change in pendulum anisotropy can be monitored precisely. Now that a systematic method of conducting pendulum experiments has been established, it is hoped that all the various experimenters will take advantage of this to develop standardized experiments allowing credible comparisons between sites and epochs. In the upcoming part II of this work, nonlinearity analysis with the help of the anisosphere will be introduced. It will be applied in retrospect to the impressive work of Maurice Allais with his paraconical pendulum. This highly nonlinear instrument was the heart of an outstanding research program conducted by a man well ahead of his time. It is hoped that the anisosphere analysis of his data will shed a new light on his experimental results.

The author is deeply indebted to Thomas J. Goodey for indefectible collaboration, very pertinent suggestions and for software development. Beneficial discussions with Jean-Bernard Deloly are gratefully acknowledged, as well as access to a draft translation of Kamerlingh Onnes' thesis by Victor O. de Haan. Finally, the original measurements reported in this work were skillfully performed by Dimitrie Olenici in his Pendularium.

\section{References}

1. M. Allais, C. R. Acad. Sci. 247, 1428 (1958)

2. M. Allais, C. R. Acad. Sci. 247, 2284 (1958)

3. M. Allais, L'Anisotropie de l'Espace (C. Juglar, Paris, 1997), p. 750

4. G.T. Jeverdan, G.I. Rusu, V.I. Antonescu, An. Univ. Iasi 7, 457, (1961)

5. I. Mihaila, N. Marcov, V. Pambuccian, M. Agop, Proc. Romanian Academy A 4, 3 (2003)

6. T.J. Goodey, A.F. Pugach, D. Olenici, J. Adv. Res. Phys. 1, 021007 (2010)

7. M. Duval, Phys. Essays 18, 53 (2005)

8. Q.-S. Wang, X.-S. Yang, C.-Z. Wu, H.-G. Guo, H.-C. Liu, C.-C. Hua, Phys. Rev. D 62, 041101 (2000)

9. T. Kuusela, Phys. Rev. D 43, 2041 (1991)

10. E.J. Saxl, M. Allen, Phys. Rev. D 3, 823 (1971)

11. A. Pugach, Int. J. Astron. Astrophys. 3, 33 (2013)

12. A. Iovane, Should the Laws of Gravitation be Reconsidered?, edited by H.A. Munera (Apeiron, Montreal, 2011), p. 175

13. T. Kapitaniak, J. Kurths, Eur. Phys. J. Special Topics 223, 609 (2014)

14. E.O. Schulz-DuBois, Am. J. Phys. 18, 173 (1970)

15. Y. Yamashita, J. Phys. Soc. Japan 85, 064002 (2016)

16. H. Kamerlingh Onnes, Ph.D. thesis, University of Groningen, NL, 1879

17. G.B. Airy, Proc. Royal Astron. Soc. XX, 121 (1851)

18. H. Poincaré, Théorie Mathématique de la Lumière, vol. II (Gauthier-Villars, Paris, 1892), Chap. XII

19. G.N. Ramachandran, S. Ramaseshan, Handbuch der Physik, Bd 5/25, edited by S. Flügge (Springer, Berlin, 1961), p. 1-217

20. R. Verreault, Z. Kristall. 136, 350 (1972)

21. H. Kamerlingh Onnes, (translation: V.O. de Haan and T. Goodey), Should the Laws of Gravitation be Reconsidered? edited by H.A. Munera (Apeiron, Montreal, 2011), p. 425

22. H. Kamerlingh Onnes, Ph.D. thesis (University of Groningen, NL, 1879)

23. R. Verreault, T.J. Goodey, D. Olenici, J.-B. Deloly, Meas. Sci. Technol. (to be published)

Open Access This article is distributed under the terms of the Creative Commons Attribution License http:// creativecommons.org/licenses/by/4.0/ which permits unrestricted use, distribution, and reproduction in any medium, provided the original author(s) and source are credited. 